\title{
Chiral-symmetry restoration in the linear sigma model at nonzero temperature and baryon density
}

\author{
Neven Bilić and Hrvoje Nikolić \\ Rudjer Bošković Institute, \\ P.O. Box 1016, 10001 Zagreb, Croatia \\ IRB-TP 229/97, hep-ph/9711513
}

May 4, 2019

\begin{abstract}
We study the chiral phase transition in the linear sigma model with 2 quark flavors and $N_{c}$ colors. One-loop calculations predict a first-order phase transition at both $\mu=0$ and $\mu \neq 0$. We also discuss the phase diagram and make a comparison with a thermal parametrization of existing heavy-ion experimental data.
\end{abstract}

\section{Introduction}

The linear sigma model, originally proposed as a model for strong nuclear interactions [回, today serves as an effective model for the low-energy (low-temperature) phase of quantum chromodynamics. The model exhibits spontaneous breaking of chiral symmetry and restoration at finite temperature. In this paper we discuss the pattern of symmetry breaking and its restoration at nonzero temperature and nonzero chemical potential at one-loop level. Some aspects of this have been extensively studied in the literature [2-12]. The phenomenological importance of the chiral transition and possible experimental signatures have recently been discussed by Harris and Müller [13]. In connection with theoretical predictions of the phase transition there still exist a certain misunderstanding and controversy. In particular, the precise value of the critical temperature is not generally agreed on [0, 6, 0, 9, 10]. As regards the order of the transition, Pisarski and Wilczek [14] have shown on general grounds that chiral models with 2 massless flavors undergo a first-order phase transition at finite temperature. In actual calculations, the mean field predicts a second-order transition [3, 12], whereas some improved mean-field results indicate a weak first-order transition [5]. Numerical simulation [15] of a three-dimensional chiral model on the lattice also confirms a first-order transition for $N_{f}=2$. We believe that in our approach we are able to clear up some of these points. In addition, we discuss the phase diagram and its relation to heavy-ion collisions.

We organize the paper as follows. In Sect. 2 we describe the thermodynamics of the model. In Sect. 3 we calculate the temperature dependence of the pion and sigma masses. 
In Sect. 4 we discuss the chiral phase transition and make comments on and a comparison with other papers. In the concluding section, Sect. 5, we summarize our results.

\section{Effective potential}

The linear sigma model of Gell-Mann and Lévy is an effective model of strong interactions described by the chirally symmetric Lagrangian (in Euclidean notation)

$$
\begin{aligned}
\mathcal{L}= & \bar{\psi}\left(\gamma_{\mu} \partial_{\mu}+g\left(\sigma+i \boldsymbol{\tau} \boldsymbol{\pi} \gamma_{5}\right)\right) \psi-\mu \bar{\psi} \gamma_{4} \psi \\
& +\frac{1}{2}(\partial \sigma)^{2}+\frac{1}{2}(\partial \boldsymbol{\pi})^{2}+\frac{m_{0}^{2}}{2}\left(\sigma^{2}+\boldsymbol{\pi}^{2}\right)+\frac{\lambda}{4}\left(\sigma^{2}+\boldsymbol{\pi}^{2}\right)^{2}
\end{aligned}
$$

The right and left fermions $\psi_{R}=\frac{1}{2}\left(1+\gamma_{5}\right) \psi, \psi_{L}=\frac{1}{2}\left(1-\gamma_{5}\right) \psi$, are assumed to constitute, respectively, the $\left(\frac{1}{2}, 0\right)$ and $\left(0, \frac{1}{2}\right)$ representation of the chiral $\mathrm{SU}(2) \times \mathrm{SU}(2)$, whereas the mesons $(\sigma, \boldsymbol{\pi})$ belong to the $\left(\frac{1}{2}, \frac{1}{2}\right)$ representation. In the original sigma model [1] the fermion field was a nucleon. We consider the fermions to be constituent quarks [7, 8] with an additional degree of freedom, "color", from the $\mathrm{SU}\left(N_{c}\right)$ local gauge group of an underlying gauge theory (QCD).

If $m_{0}^{2}<0$, the chiral symmetry will be spontaneously broken. At the classical level, the $\sigma$ and $\pi$ fields develop nonvanishing expectation values such that

$$
\langle\sigma\rangle^{2}+\langle\boldsymbol{\pi}\rangle^{2}=-\frac{m_{0}^{2}}{\lambda} \equiv f_{\pi}^{2}
$$

It is convenient to choose here

$$
\left\langle\pi_{i}\right\rangle=0, \quad\langle\sigma\rangle=f_{\pi}
$$

In order to study the thermodynamics of the model, we define the thermodynamical potential as a function of the chemical potential $\mu$ associated with baryon number density and inverse temperature $\beta=1 / T$

$$
\Omega(\beta, \mu)=-\frac{1}{\beta V} \ln Z,
$$

where the partition function $Z$ is defined as a path integral

$$
Z=\int[d \varphi] \exp \left\{-\int_{0}^{\beta} d \tau \int d^{3} x \mathcal{L}(\varphi)\right\}
$$

Here $[d \varphi]$ is an abbreviation for the integral over $\psi, \sigma, \boldsymbol{\pi}$, and $\mathcal{L}$ is given by (11). Next we introduce the saddle-point method of Frei and Patkós [16]. Our approach is similar to that of Meyer-Ortmanns and Schaefer [17] who applied the method to the chiral $\mathrm{SU}(3) \times \mathrm{SU}(3$.) We first redefine the fields

$$
\begin{aligned}
& \boldsymbol{\pi} \rightarrow \boldsymbol{\pi}+\boldsymbol{\pi}^{\prime}(x), \\
& \sigma \rightarrow \sigma+\sigma^{\prime}(x),
\end{aligned}
$$


where $\boldsymbol{\pi}^{\prime}$ and $\sigma^{\prime}$ are quantum fluctuations around the constant values $\boldsymbol{\pi}$ and $\sigma$, respectively. Next we use the transformation which quadratizes the quartic interaction

$$
\exp \left\{-\int d^{4} x \frac{\lambda}{4}\left(\sigma^{\prime 2}+\boldsymbol{\pi}^{\prime 2}\right)^{2}\right\}=\int_{c-i \infty}^{c+i \infty}[d s] \exp \left\{-\int d^{4} x\left[\frac{s}{2}\left(\sigma^{\prime 2}+\boldsymbol{\pi}^{\prime 2}\right)-\frac{s^{2}}{4 \lambda}\right]\right\} .
$$

and redefine the auxiliary field

$$
s(x) \rightarrow s+s^{\prime}(x)
$$

so that the saddle-point value $s$ maximizes the integrand. The thermodynamical potential $\Omega$ as a function of $\sigma, \boldsymbol{\pi}$ and $s$ is usually called the effective potential. Thermodynamics requires that, in thermal equilibrium, $\Omega$ should assume a minimum with respect to variations of $\boldsymbol{\pi}$ and $\sigma$. Owing to the specific form of the interaction and because of (2) and (3), we can keep $\boldsymbol{\pi}=0$ and consider $\Omega$ as a function of the two mean fields $\sigma$ and $s$. Using (6), (7) and (8) we find

$$
\Omega(\sigma, s)=\frac{\lambda}{4} \sigma^{4}+\frac{m_{0}^{2}}{2} \sigma^{2}-\frac{s^{2}}{4 \lambda}-\frac{1}{\beta V} \ln Z^{\prime}(\sigma, s),
$$

where $Z^{\prime}$ is the partition function for the shifted Lagrangian in which the quartic interaction is absent and chiral symmetry is explicitly broken:

$$
\begin{aligned}
\mathcal{L}^{\prime}= & \bar{\psi}\left(\gamma \partial+m_{F}+g\left(\sigma^{\prime}+i \boldsymbol{\tau} \boldsymbol{\pi}^{\prime} \gamma_{5}\right)\right) \psi-\mu \bar{\psi} \gamma_{4} \psi+\frac{1}{2}(\partial \boldsymbol{\pi})^{\prime 2}+\frac{1}{2}\left(\partial \sigma^{\prime}\right)^{2} \\
& +\frac{m_{\sigma}^{2}}{2} \sigma^{\prime 2}+\frac{m_{\pi}^{2}}{2} \boldsymbol{\pi}^{\prime 2}-\frac{1}{4 \lambda} s^{\prime 2}+g^{\prime} \sigma^{\prime}\left(\sigma^{\prime 2}+\boldsymbol{\pi}^{\prime 2}\right)+\frac{s^{\prime}}{2}\left(\sigma^{\prime 2}+\boldsymbol{\pi}^{\prime 2}\right)+c \sigma^{\prime}-\frac{1}{2 \lambda} s s^{\prime} .
\end{aligned}
$$

The effective masses, the trilinear coupling $g^{\prime}$ and $c$ are functions of $\sigma$ and $s$ defined as

$$
\begin{aligned}
m_{\sigma}^{2} & =m_{0}^{2}+s+3 \lambda \sigma^{2}, \quad m_{F}=g \sigma, \\
m_{\pi}^{2} & =m_{0}^{2}+s+\lambda \sigma^{2}, \quad g^{\prime}=\lambda \sigma, \\
c & =\sigma\left(m_{0}^{2}+\lambda \sigma^{2}\right) .
\end{aligned}
$$

The condition for an extremum

$$
\frac{\partial \Omega}{\partial \sigma}=0, \quad \frac{\partial \Omega}{\partial s}=0
$$

gives two equations for $\sigma$ and $s$ with solutions that will in general depend on temperature and chemical potential. A nontrivial solution $\sigma(\beta, \mu)$ will be referred to as chiral condensate.

At the classical level (neglecting the quantum and thermal fluctuations) the potential takes the form

$$
\Omega(\sigma, s)=\frac{1}{4} \lambda \sigma^{4}+\frac{m_{0}^{2}}{2} \sigma^{2}-\frac{s^{2}}{4 \lambda} .
$$

An extremum (minimum with respect to $\sigma$, maximum with respect to $s$ ) occurs at

$$
\sigma^{2}=-\frac{m_{0}^{2}}{\lambda} \equiv f_{\pi}^{2}, \quad s=0
$$

yielding

$$
m_{\pi}=0, m_{F}=g f_{\pi}, m_{\sigma}^{2}=2 \lambda f_{\pi}^{2}, g^{\prime}=\lambda f_{\pi}, c^{\prime}=0 .
$$


Thus, at the classical level, we have 3 massless pions, as it should be owing to the Goldstone theorem.

The thermal and quantum fluctuations will change the effective potential into

$$
\Omega(\sigma, s)=\frac{\lambda}{4} \sigma^{4}+\frac{m_{0}^{2}}{2} \sigma^{2}-\frac{s^{2}}{4 \lambda}+\Omega_{0}(\sigma, s)+\Omega_{I}(\sigma, s) .
$$

Here $\Omega_{I}$ contains loop corrections and $\Omega_{0}$ is the thermodynamical potential for a noninteracting gas of fermions and bosons:

$$
\begin{gathered}
\Omega_{0}=\Omega_{F}+\Omega_{\sigma}+\Omega_{\pi}, \\
\Omega_{F}=-N_{c} N_{f} \frac{1}{\beta} \sum_{l} \int \frac{d^{3} p}{(2 \pi)^{3}} \operatorname{Tr} \ln \left[\beta\left(-i \not p+m_{F}\right)\right], \\
\Omega_{\sigma}=\frac{1}{2 \beta} \sum_{n} \int \frac{d^{3} k}{(2 \pi)^{3}} \ln \left[\beta^{2}\left(k^{2}+m_{\sigma}^{2}\right)\right], \\
\Omega_{\pi}=\left(N_{f}^{2}-1\right) \frac{1}{2 \beta} \sum_{n} \int \frac{d^{3} k}{(2 \pi)^{3}} \ln \left[\beta^{2}\left(k^{2}+m_{\pi}^{2}\right)\right],
\end{gathered}
$$

where

$$
\begin{gathered}
p=\left((2 l+1) \frac{\pi}{\beta}+i \mu, \boldsymbol{p}\right), \\
k=\left(2 n \frac{\pi}{\beta}, \boldsymbol{k}\right),
\end{gathered}
$$

and the number of flavors is $N_{f}=2$. The extremum condition now reads

$$
\begin{aligned}
\frac{\partial \Omega}{\partial \sigma}= & \lambda \sigma^{3}+m_{0}^{2} \sigma+\frac{1}{\beta V} \int d^{4} x\left\{g\langle\bar{\psi}(x) \psi(x)\rangle+3 \lambda \sigma\left\langle\sigma^{\prime}(x)^{2}\right\rangle\right. \\
& \left.+\lambda \sigma\left\langle\boldsymbol{\pi}^{\prime}(x)^{2}\right\rangle+\lambda\left\langle\sigma^{\prime}(x)\left(\sigma^{\prime}(x)^{2}+\boldsymbol{\pi}^{\prime}(x)^{2}\right)\right\rangle+\left(m_{0}^{2}+3 \lambda \sigma^{2}\right)\left\langle\sigma^{\prime}(x)\right\rangle\right\}=0, \\
\frac{\partial \Omega}{\partial s}= & -\frac{s}{2 \lambda}+\frac{1}{\beta V} \int d^{4} x\left\{\frac{1}{2}\left\langle\sigma^{\prime}(x)^{2}+\boldsymbol{\pi}^{\prime}(x)^{2}\right\rangle-\frac{1}{2 \lambda}\left\langle s^{\prime}(x)\right\rangle\right\}=0 .
\end{aligned}
$$

The terms $\left\langle\sigma^{\prime}\right\rangle$ and $\left\langle s^{\prime}\right\rangle$ vanish because the quantum fluctuations take place around the true vacuum. Equations (21) and (22) become

$$
\begin{gathered}
\lambda \sigma^{3}+m_{0}^{2} \sigma-2 g N_{c} \mathcal{G}_{F}+3 \lambda \sigma\left[\mathcal{G}_{\sigma}+\mathcal{G}_{\pi}\right]+\lambda\left[\Gamma_{\sigma \sigma \sigma}+\Gamma_{\sigma \pi \pi}\right]=0, \\
-\frac{s}{2 \lambda}+\frac{1}{2} \mathcal{G}_{\sigma}+\frac{3}{2} \mathcal{G}_{\pi}=0 .
\end{gathered}
$$

Here $\mathcal{G}$ and $\Gamma$ denote thermal averages (with respect to the full partition function) over a product of two and three fields, respectively. For example,

$$
\mathcal{G}_{\sigma}=\frac{1}{\beta V} \int d^{4} x\left\langle\sigma(x)^{2}\right\rangle .
$$


This coincides with the full 2-point Green's function at $x=0$. $\mathcal{G}$ is often referred to as tadpole. Equations (23) and (24), schematically depicted in Fig. 1, state the fact that tadpoles cancel [18] also at nonzero temperature and chemical potential.

Solutions to (23) and (24) are implicit functions of $T$ and $\mu$. The tadpoles at one-loop order are given by

$$
\begin{aligned}
\mathcal{G}_{F} & =\frac{1}{\beta} \sum_{l} \int \frac{d^{3} p}{(2 \pi)^{3}} \operatorname{Tr} \frac{1}{-i \not p+m_{F}}, \\
\mathcal{G}_{\sigma, \pi} & =\frac{1}{\beta} \sum_{n} \int \frac{d^{3} k}{(2 \pi)^{3}} \frac{1}{k^{2}+m_{\sigma, \pi}^{2}}
\end{aligned}
$$

whereas the three-point functions $\Gamma_{\sigma \sigma \sigma}$ and $\Gamma_{\sigma \pi \pi}$ contribute at two-loop (and higher) order. The masses in (26) depend on $\sigma$ and $s$ through (11). Equation (23) has, apart from $\sigma=0$, a nontrivial solution $\sigma(\beta, \mu)$ that no longer equals $f_{\pi}$. Dividing it by $\lambda \sigma$, this equation at one-loop order may be written as

$$
\begin{aligned}
\sigma^{2}= & f_{\pi}^{2}+4 \frac{2 g^{2}}{\lambda} N_{c} \frac{1}{\beta} \sum \int \frac{d^{3} p}{(2 \pi)^{3}} \frac{1}{p^{2}+m_{F}^{2}} \\
& -3 \frac{1}{\beta} \sum \int \frac{d^{3} k}{(2 \pi)^{3}} \frac{1}{k^{2}+m_{\sigma}^{2}}-3 \frac{1}{\beta} \sum \int \frac{d^{3} k}{(2 \pi)^{3}} \frac{1}{k^{2}+m_{\pi}^{2}}
\end{aligned}
$$

where we have replaced $-m_{0}^{2} / \lambda=f_{\pi}^{2}$ from (14) and used $m_{F}=g \sigma$. Similarly, (24) becomes

$$
s=\lambda \frac{1}{\beta} \sum \int \frac{d^{3} k}{(2 \pi)^{3}} \frac{1}{k^{2}+m_{\sigma}^{2}}+3 \lambda \frac{1}{\beta} \sum \int \frac{d^{3} k}{(2 \pi)^{3}} \frac{1}{k^{2}+m_{\pi}^{2}} .
$$

We can separate the finite $T$ and $\mu$ part of $\mathcal{G}$ as usual [19]:

$$
\mathcal{G}_{F}=4 \int \frac{d^{4} q}{(2 \pi)^{4}} \frac{m_{F}}{q^{2}+m_{F}^{2}}-4 \int \frac{d^{3} q}{(2 \pi)^{3}} \frac{m_{F}}{2 \omega_{F}} n_{F}\left(\omega_{F}\right),
$$

and

$$
\mathcal{G}_{\sigma, \pi}=\int \frac{d^{4} q}{(2 \pi)^{4}} \frac{1}{q^{2}+m_{\sigma, \pi}^{2}}+\int \frac{d^{3} q}{(2 \pi)^{3}} \frac{1}{\omega_{\sigma, \pi}} n_{B}\left(\omega_{\sigma, \pi}\right)
$$

where

$$
\begin{gathered}
\omega_{F}^{2}=\boldsymbol{q}^{2}+m_{F}^{2}, \quad \omega_{\sigma, \pi}^{2}=\boldsymbol{q}^{2}+m_{\sigma, \pi}^{2}, \\
n_{F}(\omega)=\frac{1}{e^{\beta(\omega-\mu)}+1}+\frac{1}{e^{\beta(\omega+\mu)}+1}, \\
n_{B}(\omega)=\frac{1}{e^{\beta \omega}-1} .
\end{gathered}
$$

The infinite part in (29) and (30) is the usual $T=\mu=0$ tadpole that is absorbed in the tadpole cancellation at the tree level. Therefore, we can write our equations by retaining the $T$ - and $\mu$-dependent pieces only. Equations (27) and (28) finally read 


$$
\begin{aligned}
\sigma^{2}= & f_{\pi}^{2}-\frac{8 g^{2}}{\lambda} N_{c} \int \frac{d^{3} q}{(2 \pi)^{3}} \frac{1}{2 \omega_{F}} n_{F}\left(\omega_{F}\right) \\
& -3 \int \frac{d^{3} q}{(2 \pi)^{3}} \frac{1}{\omega_{\sigma}} n_{B}\left(\omega_{\sigma}\right)-3 \int \frac{d^{3} q}{(2 \pi)^{3}} \frac{1}{\omega_{\pi}} n_{B}\left(\omega_{\pi}\right), \\
s= & \lambda \int \frac{d^{3} q}{(2 \pi)^{3}} \frac{1}{\omega_{\sigma}} n_{B}\left(\omega_{\sigma}\right)+3 \lambda \int \frac{d^{3} q}{(2 \pi)^{3}} \frac{1}{\omega_{\pi}} n_{B}\left(\omega_{\pi}\right),
\end{aligned}
$$

where the right-hand sides depend on $\sigma$ and $s$ through the masses. These equations have been derived from the effective potential (16) in which the loop corrections $\Omega_{I}$ have been neglected. This approximation corresponds to the leading order in the $1 / N$ expansion, where $N$ is the number of scalar fields [17]. In our case, $N=4$.

A straightforward approach to solving (34) and (35) leads to problems with a complex effective potential [17. It may easily be seen that a direct use of (11) leads necessarily to complex solutions. From (34), (35) with (11) one finds

$$
m_{\pi}^{2}=-8 g^{2} N_{c} \int \frac{d^{3} q}{(2 \pi)^{3}} \frac{1}{2 \omega_{F}} n_{F}\left(\omega_{F}\right)-2 \lambda \int \frac{d^{3} q}{(2 \pi)^{3}} \frac{1}{\omega_{\sigma}} n_{B}\left(\omega_{\sigma}\right) .
$$

This means that $m_{\pi}^{2}$ is either negative or complex. In both cases it implies a complex $\sigma$. In the following sections we show how a consistent inclusion of one-loop self- energy corrections removes this problem.

\section{$3 \quad$ Effective meson masses}

At first sight it seems that the $T, \mu$ dependence of $\sigma$ and $s$ is not consistent with the Goldstone theorem since

$$
m_{0}^{2}+s(\beta, \mu)+\lambda \sigma^{2}(\beta, \mu) \neq 0 .
$$

However, the $m_{\pi}^{2}$ must also include the $T$ - and $\mu$-dependent pieces coming from the one-loop (and higher) order self-energy diagrams (Fig. 2a):

$$
m_{\pi}^{2}=m_{0}^{2}+s(\beta, \mu)+\lambda \sigma^{2}(\beta, \mu)+\Pi_{\pi}(\beta, \mu)
$$

where (at one-loop order)

$$
\begin{aligned}
\Pi_{\pi}= & -8 N_{c} g^{2} \frac{1}{\beta} \sum \int \frac{d^{3} p}{(2 \pi)^{3}} \frac{1}{p^{2}+m_{F}^{2}}-4 g^{2} \frac{1}{\beta} \sum \int \frac{d^{3} k}{(2 \pi)^{3}} \frac{1}{k^{2}+m_{\sigma}^{2}} \frac{1}{k^{2}+m_{\pi}^{2}} \\
& -\frac{1}{\beta} \sum \int \frac{d^{3} k}{(2 \pi)^{3}} \frac{1}{k^{2}+m_{\pi}^{2}}(-2 \lambda),
\end{aligned}
$$

with $p$ and $k$ defined as in (19) and (20), respectively. The $s$-field appears in the Lagrangian without a kinetic term and its propagator is simply $1 / m_{s}^{2}=-2 \lambda$. 
The combined contribution of the $\sigma$-tadpoles (27) and the $s$-tadpoles (28) is given by

$$
\begin{aligned}
m_{0}^{2}+s+\lambda \sigma^{2}= & \underbrace{m_{0}^{2}+\lambda f_{\pi}^{2}}_{=0}+8 g^{2} N_{c} \frac{1}{\beta} \sum \int \frac{d^{3} p}{(2 \pi)^{3}} \frac{1}{p^{2}+m_{F}^{2}} \\
& -2 \lambda \frac{1}{\beta} \sum \int \frac{d^{3} k}{(2 \pi)^{3}} \frac{1}{k^{2}+m_{\sigma}^{2}} .
\end{aligned}
$$

Combining (39) and (40), we see that the fermion parts cancel immediately, whereas the boson parts cancel provided the relation

$$
m_{\sigma}^{2}-m_{\pi}^{2}=2 \lambda \sigma^{2}
$$

which holds trivially at $T=\mu=0$, also holds at $T, \mu \leq T_{c}, \mu_{c}$. Thus, the consistency with the Goldstone theorem requires that the relation

$$
m_{\sigma}^{2}=2 \lambda \sigma^{2}
$$

should also hold at $T, \mu \leq T_{c}, \mu_{c}$. Indeed, we shall shortly demonstrate that it works at one-loop level.

We do not agree here with Larsen [5] who obtained $m_{\pi} \neq 0$ even in the symmetry-broken phase, thus violating the Goldstone theorem. The reason is that he assumed $m_{\pi} \neq 0$ in the propagators in the one-loop self-energy diagrams. If $m_{\pi}=0$ at the tree level, then the mass corrections would be of order $\lambda$ or higher. This would in turn yield self-energy corrections of order $\lambda^{2}$, which would then also require the inclusion of two-loop diagrams. In other words, it is not consistent with one-loop calculations to include corrections to the tree-level masses in the propagator.

Similarly as for the pion mass, for the sigma mass we have

$$
m_{\sigma}^{2}=m_{0}^{2}+s(\beta, \mu)+3 \lambda \sigma^{2}(\beta, \mu)+\Pi_{\sigma}(\beta, \mu),
$$

where the self-energy of the $\sigma$ particle (Fig. $2 \mathrm{~b}$ ) is given by

$$
\begin{aligned}
\Pi_{\sigma}= & -8 g^{2} N_{c} \frac{1}{\beta} \sum \int \frac{d^{3} p}{(2 \pi)^{3}}\left(\frac{1}{p^{2}+m_{F}^{2}}-\frac{2 m_{F}^{2}}{\left(p^{2}+m_{F}^{2}\right)^{2}}\right) \\
& -3 \cdot 6 g^{\prime 2} \frac{1}{\beta} \sum \int \frac{d^{3} k}{(2 \pi)^{3}} \frac{1}{\left(k^{2}+m_{\sigma}^{2}\right)^{2}}-2 \cdot 3 g^{\prime 2} \frac{1}{\beta} \sum \int \frac{d^{3} k}{(2 \pi)^{3}} \frac{1}{\left(k^{2}+m_{\pi}^{2}\right)^{2}} \\
& -\frac{1}{\beta} \sum \int \frac{d^{3} k}{(2 \pi)^{3}} \frac{1}{k^{2}+m_{\sigma}^{2}}(-2 \lambda) .
\end{aligned}
$$

This, together with the tadpole part

$$
\begin{aligned}
m_{0}^{2}+s+3 \lambda \sigma^{2}= & \underbrace{m_{0}^{2}+3 \lambda f_{\pi}^{2}}_{2 \lambda f_{\pi}^{2}}+24 g^{2} N_{c} \frac{1}{\beta} \sum \int \frac{d^{3} p}{(2 \pi)^{3}} \frac{1}{p^{2}+m_{F}^{2}} \\
& -8 \lambda \frac{1}{\beta} \sum \int \frac{d^{3} k}{(2 \pi)^{3}} \frac{1}{k^{2}+m_{\sigma}^{2}}-6 \lambda \frac{1}{\beta} \sum \int \frac{d^{3} k}{(2 \pi)^{3}} \frac{1}{k^{2}+m_{\pi}^{2}},
\end{aligned}
$$


gives

$$
\begin{aligned}
m_{\sigma}^{2}= & 2 \lambda f_{\pi}^{2}+2 \cdot 8 g^{2} N_{c} \frac{1}{\beta} \sum \int \frac{d^{3} p}{(2 \pi)^{3}} \frac{1}{p^{2}+m_{F}^{2}} \\
& -2 \cdot 3 \lambda \frac{1}{\beta} \sum \int \frac{d^{3} k}{(2 \pi)^{3}} \frac{1}{k^{2}+m_{\sigma}^{2}}-2 \cdot 3 \lambda \frac{1}{\beta} \sum \int \frac{d^{3} k}{(2 \pi)^{3}} \frac{1}{\left(k^{2}+m_{\pi}^{2}\right)}+\ldots
\end{aligned}
$$

where ... denotes terms of higher order in $\lambda$ and $g^{2}$. Combining this equation with (27), we recover (42). Thus, the thermal corrections at one-loop level do not alter the tree-level equation for the effective sigma mass.

\section{Chiral restoration transition}

From the analysis in the preceding section we see that the $s$-dependence of the $m_{\sigma}$ and $m_{\pi}$ is removed owing to the one-loop self-energy corrections. Equations (34), (35) in which we put

$$
\begin{aligned}
& m_{\pi}=0, \\
& m_{\sigma}^{2}=2 \lambda \sigma^{2}, \\
& m_{F}=g \sigma,
\end{aligned}
$$

decouple and we are able to determine the $T$ and $\mu$ dependence of the chiral condensate by solving (34) only. If one compares our approach with the standard one where the quartic interaction is kept, one may easily check that the self-energy diagrams with internal $s$-lines plus the contribution of $s$-tadpoles precisely equals the contribution of the loops with the quartic vertex. In this way, the saddle-point method [16, 17] becomes equivalent to the standard approach.

For $\mu=0$, we find the solution numerically as a function of $T$ depicted in Fig. 3. As input parameters we choose the constituent quark mass $m_{F}=340 \mathrm{MeV}$ and the sigma mass $m_{\sigma}=1 \mathrm{GeV}$. The solution indicates a first-order phase transition. The point where the curve crosses the $T$ axis is not the point of the actual phase transition. We refer to it as "critical" point, having in mind that it would be a critical point if the phase transition were second order. The actual transition takes place at the point where the two minima of the effective potential at $\sigma=0$ and $\sigma\left(T_{c}\right)$ are leveled. Hence, the transition temperature $T_{c}$ is determined by requiring

$$
\Omega\left(\sigma, T_{c}\right)=\Omega\left(0, T_{c}\right) .
$$

The solution is particularly simple in the neighborhood of the "critical" point $T_{c}^{\prime}$ since in this case we can expand the integrands around $\sigma=0$ and perform the integrals analytically 《19. We find

$$
\begin{aligned}
\sigma^{2}= & f_{\pi}^{2}-\left[\frac{2 g^{2}}{\lambda} N_{c} \frac{T^{2}}{6}+\frac{T^{2}}{2}\right]+\frac{3 \sqrt{2 \lambda}}{4 \pi} \sigma T \\
& +\sigma^{2}\left[\left(\frac{\gamma}{2}-\frac{1}{4}\right)\left(\frac{3 \lambda}{2 \pi^{2}}-\frac{2 g^{4} N_{c}}{\lambda \pi^{2}}\right)+\frac{3 \lambda}{4 \pi^{2}} \ln \frac{\sqrt{2 \lambda} \sigma}{4 \pi T}-\frac{g^{4} N_{c}}{\lambda \pi^{2}} \ln \frac{g \sigma}{\pi T}\right]+\mathcal{O}\left(\sigma^{3}\right),
\end{aligned}
$$


where $\gamma=0.5772 \ldots$ is Euler's constant. At the "critical" temperature, $\sigma\left(T_{c}^{\prime}\right)=0$, which gives

$$
\frac{T_{c}^{\prime 2}}{f_{\pi}^{2}}=\frac{6 \lambda}{2 g^{2} N_{c}+3 \lambda} .
$$

If we had taken the Nambu-Jona-Lasigno relation $m_{\sigma}=2 m_{F}$ [20], which is exact in the $N_{c} \rightarrow \infty$ limit [21], we would have obtained $T_{c}^{\prime}=f_{\pi}$. We stress again that $T_{c}^{\prime}$ only approximates the actual transition temperature $T_{c}$ to be determined from the condition (48).

If we put $N_{c}=1$ in (50) our result agrees with that of Anand et al. [4] who considered a similar model for nuclear matter in Walecka's mean-field approach. Our result (50) also agrees with Bochkarev and Kapusta [12] if we put $N_{c}=0$. However, some of the existing calculations that include fermions [2, 7, 6, 9] disagree with our result for the following reasons. Ram Mohan [2], and subsequently Contreras and Loewe [7] did not properly account for the contribution of antifermions. As a consequence, they obtained fewer (by a factor of two, apart from color) fermionic degrees of freedom, which in turn yielded a larger estimate for the $T_{c}$ [9]. The reason for disagreement with Cleymans, Kocić and Scadron [6] is twofold. First, in their calculation of the sigma mass they did not include all the relevant self-energy diagrams (Fig. 22). Second, the sign of the fermionic contribution to the sigma mass is wrong. This led again to a larger $T_{c}$ in terms of $f_{\pi}$. The agreement of their estimate of the critical temperature with the estimates based on a single meson-loop diagram [1] and on the Nambu-Jona-Lasigno model [10] is only accidental.

The calculations at nonzero chemical potential are similar. We present our results in Fig. 4. The chiral condensate $\sigma$ is plotted as a function of temperature for fixed $\mu$ (160 and $350 \mathrm{MeV}$; upper two plots) and as a function of chemical potential for fixed $T$ ( 0 and $50 \mathrm{MeV}$; lower two plots). The transition remains first order and the critical temperature decreases with $\mu$ as expected.

In Fig. 5 we plot the phase diagram of nuclear matter and compare it with the thermal parametrization of recent heavy-ion collision data. The baryonic chemical potential is related to the quark chemical potential as $\mu_{B}=3 \mu$. The phase boundary between the chirally symmetric and broken phases (solid line) appears to be very close to the expected phase boundary between hadron resonance gas and quark-gluon plasma with a bag constant [13]. The diagram indicates that the chiral transition might slightly precede the deconfinement. The points with error bars show the freeze-out values of $T$ and $\mu$ deduced from AGS [22, 23, 24 and SPS [25] data with flow.

In Fig. 6 the phase diagram is represented in terms of temperature and baryon density. The solid line separating the chirally symmetric and broken phases shows how the transition temperature depends on baryon density.

Similarly to the chiral condensate, the effective masses will have a discontinuity at $T_{c}$. Above the critical line in Fig. 5 the symmetry is restored and the $\sigma$-tadpoles vanish from the theory. The $T$ and $\mu$ dependence of the meson mass $m_{M}=m_{\pi}=m_{\sigma}$ is determined by the proper self-energy diagrams and $s$-tadpoles:

$$
m_{M}^{2}=m_{0}^{2}+s(\beta, \mu)+\Pi_{\pi}(\beta, \mu) .
$$

Here $s(\beta, \mu)$ and $\Pi_{\pi}(\beta, \mu)$ may be calculated at lowest order in $\lambda$ and $g^{2}$ using (28) and (39) in which the propagator masses and $g^{\prime}$ are set to zero. In particular, for $\mu=0$ and above $T_{c}$ 
we find the following expression for the meson mass

$$
m_{M}^{2}=\left(\frac{\lambda}{2}+\frac{N_{c}}{3} g^{2}\right)\left(T^{2}-T_{c}^{\prime 2}\right) .
$$

\section{Conclusion}

We have shown that the usual mean-field pattern of symmetry breaking and restoration gives a consistent picture in the $\sigma$-model at the one-loop order. We have shown that the saddle-point method [16, 17] is equivalent to a standard approach [2, 4, 12] if the selfenergy loop corrections are included. The phase transition is predicted to be first order, in agreement with the analysis of Pisarski and Wilczek [14]. The chiral phase boundary in a $\left(T, \mu_{B}\right)$ plot (Fig. 5) is close to the phase boundary between hadron gas and quarkgluon plasma. The thermal parametrization of existing experimental data compared with the chiral phase diagram indicates that the nuclear matter produced in heavy-ion collisions is close to or slightly above the chiral-phase-transition line. It is therefore conceivable that the present and future heavy-ion experiments may observe effects of the chiral transition. A more sophisticated analysis of the data is needed in order to observe possible signatures

near the critical density, such as a rapid change of the meson mass and width or abnormal production ratios of charged to neutral pions [13].

\section{Acknowledgement}

NB would like to thank J. Cleymans, D. Lurié and M. Scadron for useful discussions.

\section{References}

[1] M. Gell-Mann and M. Lévy, Nuovo Cimento 16 (1960) 705

[2] L. R. Ram Mohan, Phys. Rev. D14 (1976) 2670

[3] G. Baym and G. Grinstein Phys. Rev. D15 (1977) 2897

[4] J. D. Anand, R. Basu, S. N. Biswas, A. Goyal and S. K. Sony, Phys. Rev. D34 (1986) 2133

[5] A. Larsen, Z. Phys. C33 (1986) 291

[6] J. Cleymans, A. Kocić and M. D. Scadron, Phys. Rev. D39 (1989) 323

[7] S. Contreras and M. Loewe, Int. J. Mod. Phys. A5 (1990) 2297

[8] A. Gocksch, Phys. Rev. Lett. 67 (1991) 1701

[9] D. Lurié and G.B. Tupper, Phys. Rev. D47 (1993) 3580

[10] D. Bilić, J. Cleymans and M. D. Scadron, Int. J. Mod. Phys. A10 (1995) 1169 
[11] R. Baier, M. Dirks and O. Kober, Phys. Rev. D54 (1996) 2222

[12] A. Bochkarev and J. Kapusta, Phys. Rev. D54 (1996) 4066

[13] J. W. Harris and B. Müller, Ann. Rev. Nucl. Part. Sci. 46 (1996) 71

[14] R. D. Pisarski and F. Wilczek, Phys. Rev. D29 (1984) 3386

[15] H. Gausterer and S. Sanielevici, Phys. Lett. b209 (1988) 533

[16] Z. Frei and A. Patkós, Phys. Lett. b247 (1990) 381

[17] H. Meyer-Ortmanns and B.-J. Sshaefer, Phys. Rev. D53 (1996) 6586

[18] J.-L. Gervais and B. W. Lee, Nucl. Phys. b12 (1969) 627

[19] J.I. Kapusta, Finite-temperature field theory, Cambridge University Press, Cambridge, UK (1989)

[20] Y. Nambu and G. Jona-Lasinio, Phys. Rev. 122 (1961) 345

[21] P.M. Fishbane, Re.E. Norton and T.N. Truong, Phys. Rev. D46 (1992) 1768

[22] P. Braun-Munzinger, J. Stachel, J. P. Wessels and N. Xu, Phys. Lett. b344 (1995) 43

[23] J. Letessier, J. Rafelski and A. Tounsi Phys. Lett. b328 (1994) 499

[24] J. Rafelski and M. Danos, Phys. Rev. C50 (1994) 1684

[25] P. Braun-Munzinger, J. Stachel, J. P. Wessels and N. Xu, Phys. Lett. b365 (1996) 1 
Figure 1: Schematic representation of (23) and (24)

Figure 2: One-loop self-energy diagrams contributing to (a) pion and (b) sigma masses.

Figure 3: Chiral condensate as a function of temperature at $\mu=0$. The dashed line corresponds to a physically unstable solution. $T_{c}$ is the temperature of the first-order phase transition.

Figure 4: Chiral condensate at nonzero baryon density. $\sigma, T$ and $\mu$ are in $\mathrm{MeV}$.

Figure 5: Phase diagram as a plot of temperature versus baryon chemical potential. The solid line separates the chirally broken (inside) and chirally symmetric (outside) phases. The expected hadron gas - quark-gluon plasma boundary is located between the two dashed lines.

Figure 6: Phase diagram as a plot of temperature versus baryon density. 

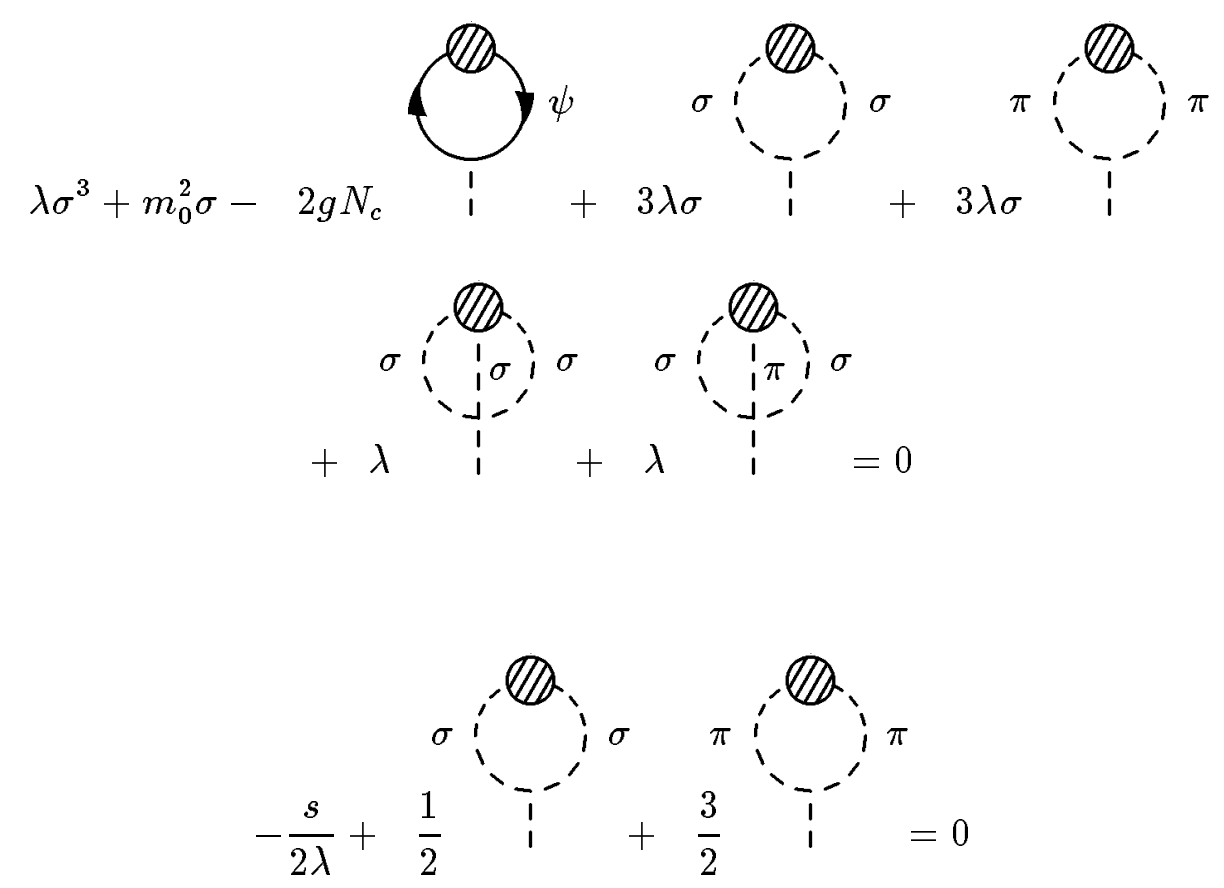

Fig.1 

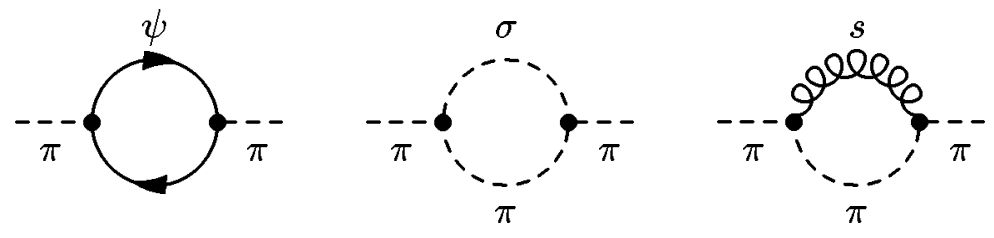

(a)
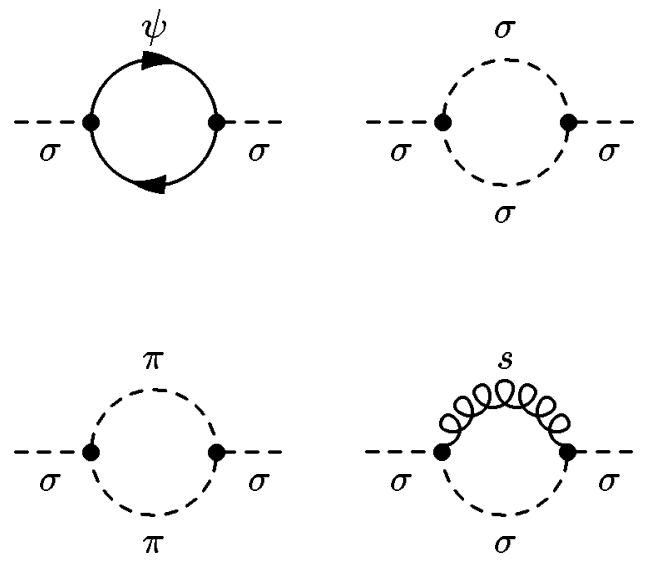

Fig.2 


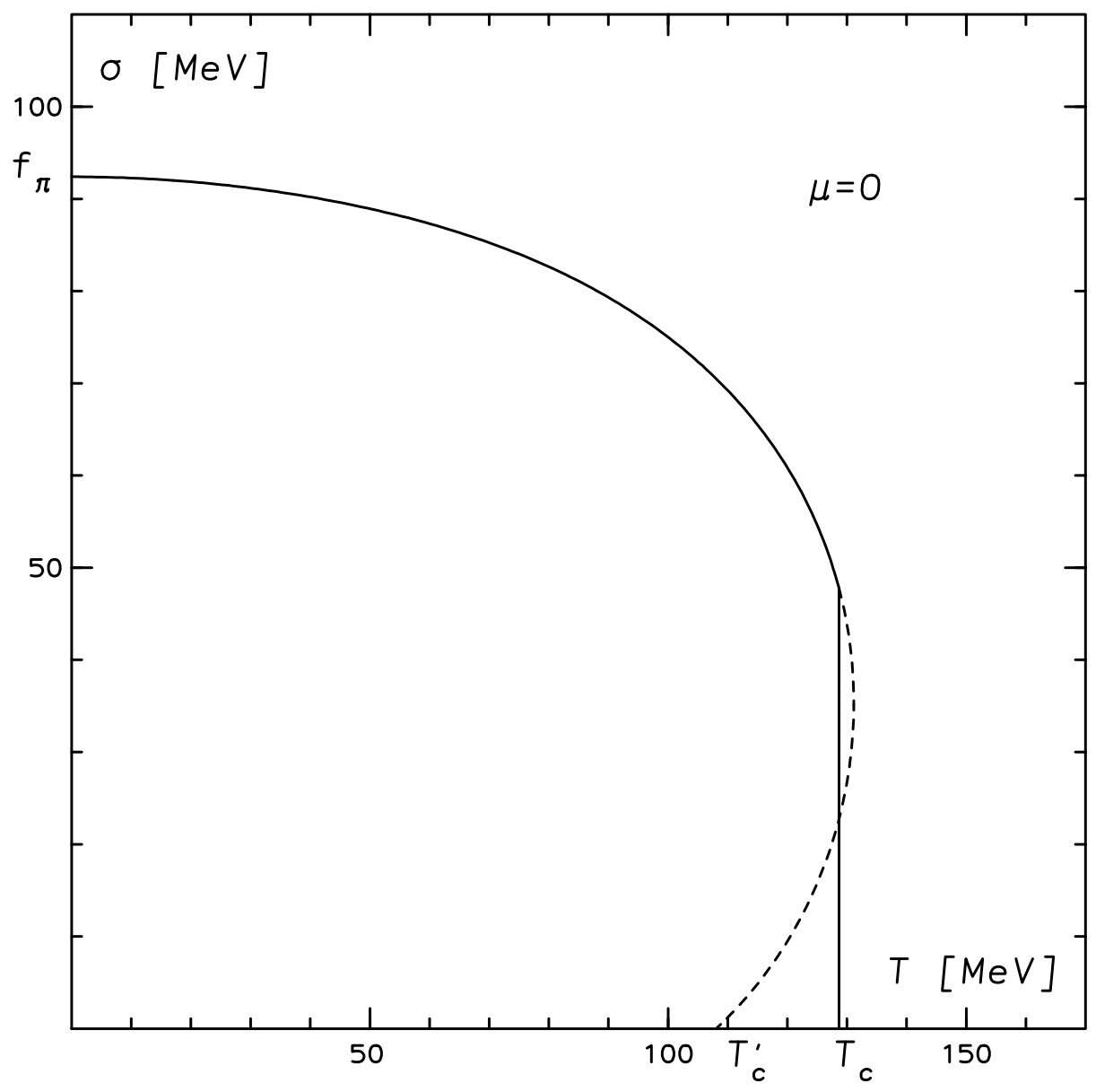

Fig. 3 

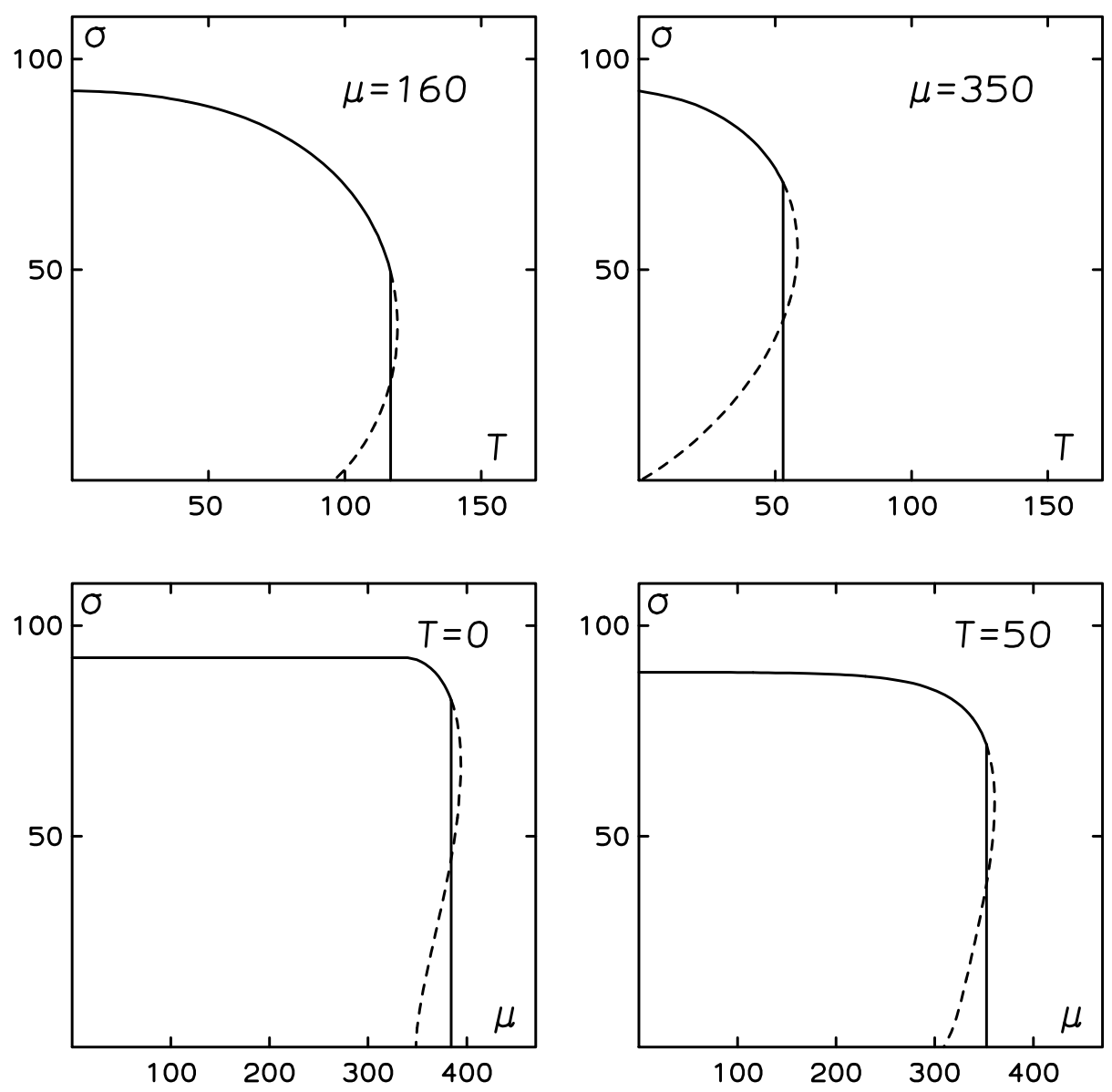

Fig. 4 


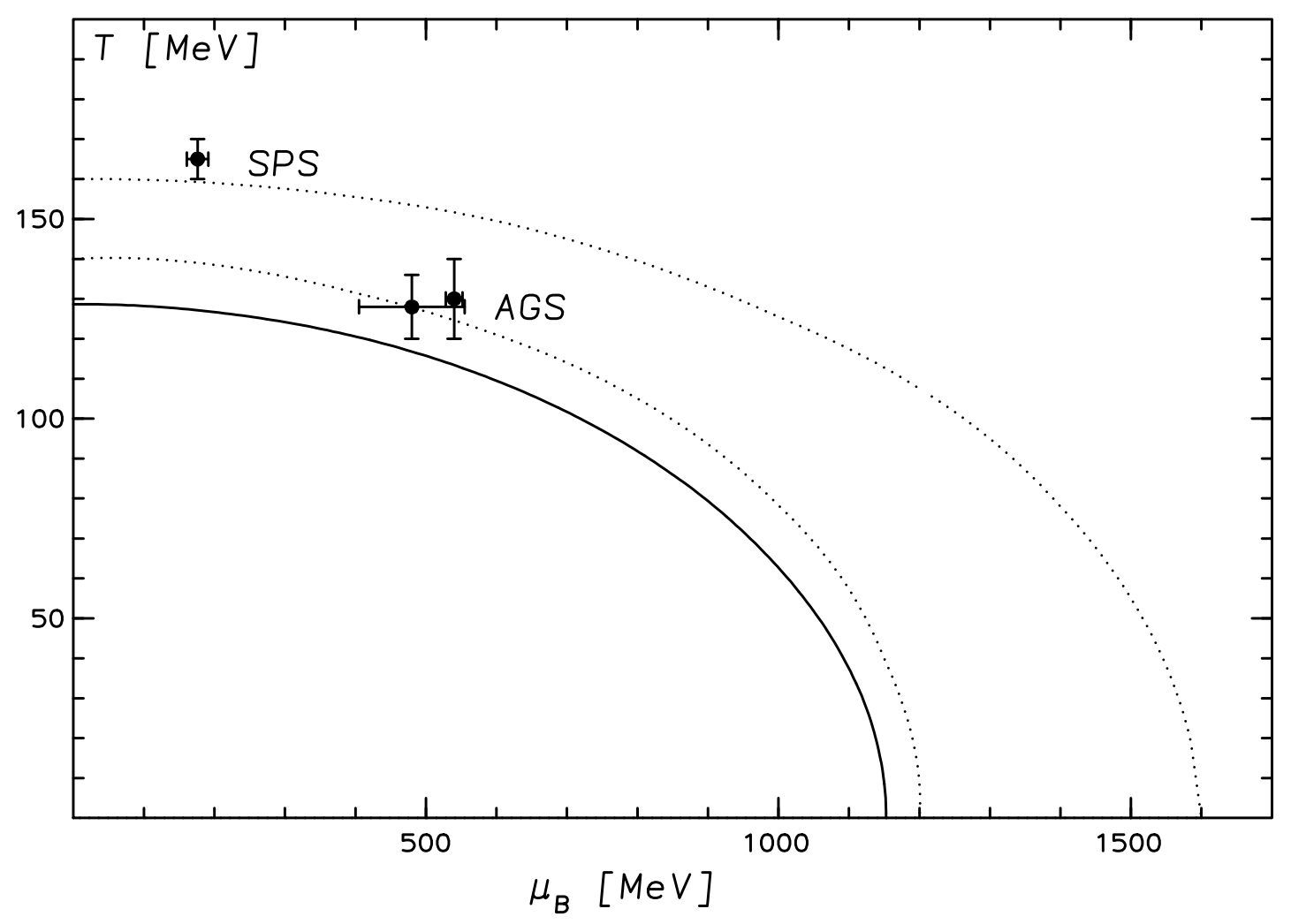

Fig. 5 


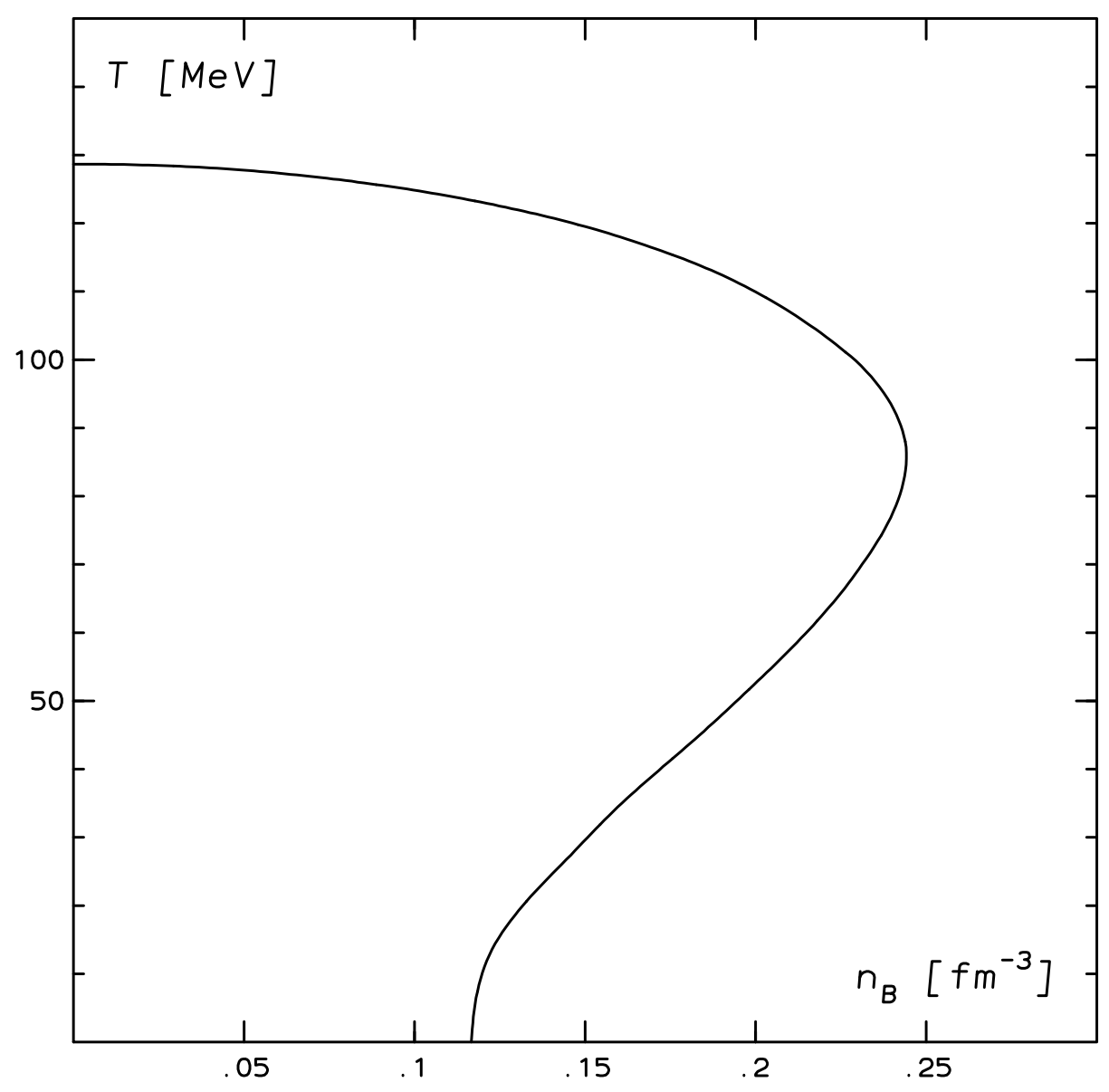

Fig. 6 